Orthopäde 2018 · 47:975-976

https://doi.org/10.1007/s00132-018-03658-6

Online publiziert: 9. November 2018

(c) Springer Medizin Verlag $\mathrm{GmbH}$, ein Teil von Springer Nature 2018

CrossMark

\title{
V. Ewerbeck
}

Zentrum für Orthopädie, Unfallchirurgie und Paraplegiologie, Universitätsklinikum Heidelberg Heidelberg, Deutschland

\section{Der Orthopäde ist kein Publikationssupermarkt des Fachs}

sein. Genau dies gewährleistet das Konzept unserer Zeitschrift, wie Sie es seit vielen Jahren kennen. Das gilt sowohl für die Themenhefte, als auch die Hefte wie dieses, welches sich ausschließlich Originalien widmet, die sich - jede für sich naturgemäß nur mit einer einzigen wissenschaftlichen Fragestellung beschäftigen können. In der Summe und über die Zeit gelingt es indessen sehr wohl, diesen breiten Überblick über unsere Themenauswahl zu erhalten.

In diesem Heft gibt es drei Publikationen, die sich mit Themen der Behandlungen von Wirbelsäulenerkrankungen beschäftigen, drei weitere zum Thema der Gelenkersatzchirurgie und deren Umfeld, unter anderem auch der präoperativen konservativen Therapie, einen Beitrag zur Infektiologie und einen weiteren Beitrag zu einem Thema aus der Osteologie im Grenzbereich zur Onkologie (Morbus Gorham-Stout).

\section{I) Die Themenschwerpunkte in diesem Heft spiegeln die Versorgungszahlen in Deutschland wieder}

Die Themenschwerpunkte in diesem Heft, nämlich die Wirbelsäulenchirurgie und die Endoprothetik, spiegeln ein Stück weit die Versorgungszahlen in Deutschland wieder, die eben diesen Schwerpunkt auch haben. Ob die so bleiben werden, sei dahingestellt. Eine wissenschaftliche Fachzeitschrift kann auch die Aufgabe haben, Entwicklungen und Trends kenntlich $\mathrm{zu}$ machen, und auf sogenannte mögliche „Gamechanger“ frühzeitig aufmerksam zu machen. Sie kündigen sich stets durch Publikationen neuer wissenschaftlicher Erkenntnisse an, von denen sich eine als bahnbrechend erweisen kann. Es ist nicht sicher, ob die Versorgungsrealität, wie sie sich zum gegenwärtigen Zeitpunkt in Deutschland darstellt, auch in Zukunft bleibt wie sie ist. Wir wissen nicht, wie dick das Eis ist, auf dem sich der hoch selektiv tätige Endoprothetiker, der ausschließlich elektiv tätige Wirbelsäulenchirurg bewegt. Andere Fachgebiete, wie die Viszeralchirurgie, die Gefäßchirurgie oder die Rheumatologie, können ein Lied davon singen, welch dramatischen Einfluss der wissenschaftliche Fortschritt auf das Spektrum ihres Fachs, die zur Verfügung stehenden Methoden und den beruflichen Alltag ihrer Ärztinnen und Ärzte gehabt hat.

Ein weiteres spannendes Thema wird Ihnen nach Durchsicht dieses Heftes sicher ebenfalls auffallen:

Wie verträgt sich die Internationalisierung unserer Zeitschrift mit unserem Anspruch, in allererster Linie für die Bedürfnisse unserer deutschsprachigen Leserschaft zu publizieren? Die Themenschwerpunkte dieses Heftes spiegeln - wie erwähnt - zwar die Versorgungsrealität in Deutschland wieder, jedoch stammen alle wissenschaftlichen Originalbeiträge zum Thema der Wirbelsäulenchirurgie dieses Heftes aus China. Wissenschaftliche Erkenntnisse, wie sie in diesen Beiträgen publiziert werden, sind nicht abhängig von der Versorgungsrealität, und somit auch nicht vom Land, aus dem diese Beiträge 
stammen. Insofern ist diese Art von Wissenschaft systemunabhängig. Für die Versorgungsforschung gilt das nicht. Dies wird aus dem Beitrag von T. Lange und Mitarbeitern zur Verordnungshäufigkeit physikalischer Therapien und Analgetika vor dem Einsatz einer Hüftbeziehungsweise Kniegelenksendoprothese in Deutschland unmittelbar deutlich. Es bleibt dabei, dass Der Orthopäde ein deutschsprachiges Organ ist und dennoch internationale Beiträge einwerben will und muss. Wir wissen, dass wir uns in der Konkurrenz der höherspezialisierten Fachzeitschriften befinden. Genau in diesem Wissen scheint mir die Breite unseres Konzepts ein Alleinstellungsmerkmal, und damit vorteilhaft zu sein. Die Frage nach der Sinnhaftigkeit von Spezialisten und Generalisten ist beantwortet. Benötigt werden nicht Spezialisten oder Generalisten, sondern Spezialisten und Generalisten. In diesem Sinne: „Der Orthopäde ist ein international angesehenes Publikationsorgan und widmet sich allen Aspekten der Orthopädie." Mit einem Supermarkt hat das nichts zu tun.

Ich wünsche Ihnen eine spannende Lektüre dieses Heftes und verbleibe

mit besten kollegialen Grüßen

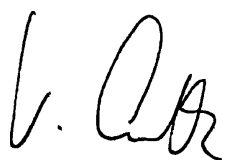

Professor Dr. med. V. Ewerbeck

Editor in Chief

Originalarbeiten

\section{Korrespondenzadresse}

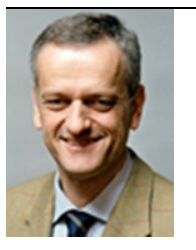

Prof. Dr. med. V. Ewerbeck Zentrum für Orthopädie, Unfallchirurgie und Paraplegiologie, Universitätsklinikum Heidelberg

Schlierbacher Landstr. 200a, 69118 Heidelberg,

Deutschland Volker.Ewerbeck@med.uniheidelberg.de

Interessenkonflikt. V. Ewerbeck gibt an, dass kein Interessenkonflikt besteht.

\section{Fachnachrichten}

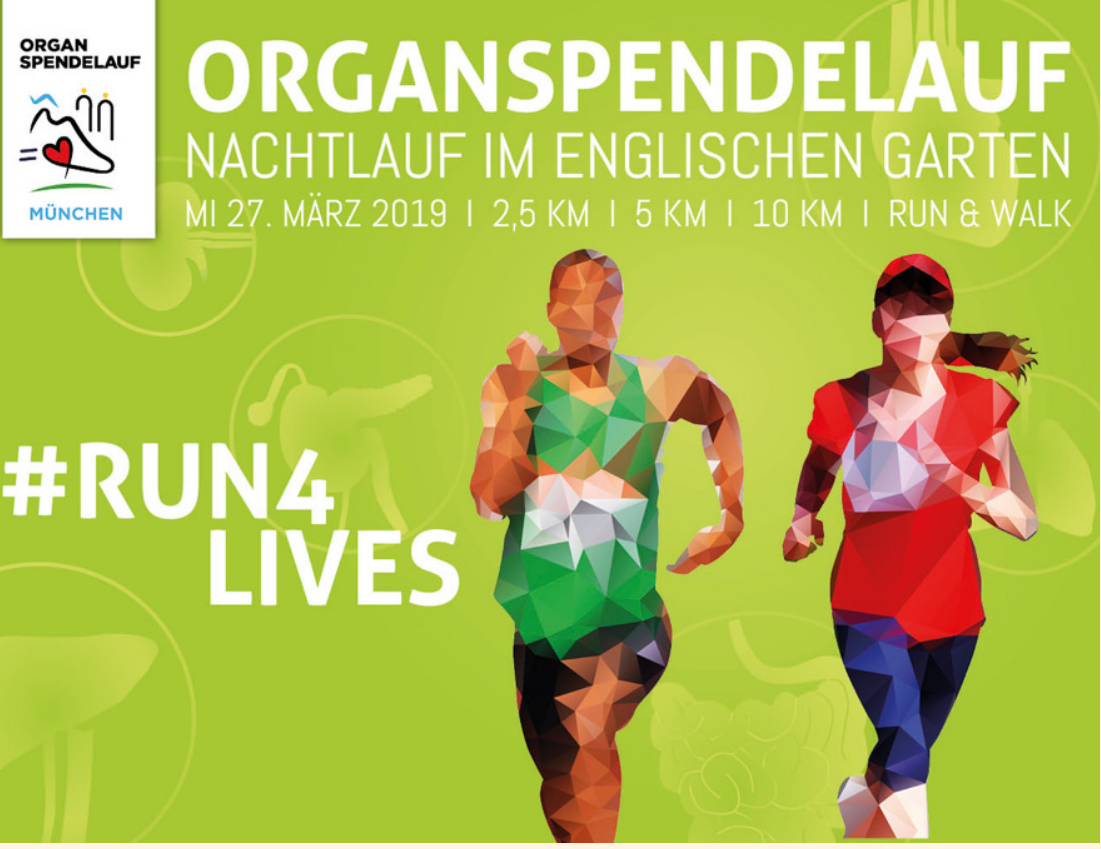

\section{Laufen rettet Leben}

\section{Organspendelauf in München am 27. März 2019}

Am Mittwoch, dem 27. März 2019 startet wieder der alljährliche Organspendelauf in München, ein fester Programmpunkt beim jährlich stattfindenden Kongress der Deutschen Gesellschaft für Chirurgie (DGCH). Eine Neuerung: Der Lauf im Englischen Garten ist erstmals offen für Jedermann und erfährt eine breite Unterstützung durch Sponsoren und Spender. Das Organisationsteam um Prof. Dr. Matthias Anthuber möchte so die Organspende noch mehr in den Fokus der Öffentlichkeit rücken. Gleichzeitig werden Spenden gesammelt für gemeinnützige Organisationen und Projekte, die sich für Organtransplantationen einsetzen.

Anthuber, aktueller Präsident der DGCH und verantwortlich für die Organisation von Kongress und Lauf, möchte das Thema Organspende und Transplantation zu einem zentralen Element beim Deutschen Chirurgenkongress 2019 machen. „Mit dem Lauf möchten wir informieren und Aufmerksamkeit wecken", sagt der Chefarzt der Klinik für Allgemein-, Viszeral- und Transplantationschirurgie am Klinikum Augsburg. Sein Ziel: Möglichst viele Menschen sollen sich mit Fragen zur Organspende auseinandersetzen und eine persönliche Entscheidung treffen wie auch immer diese ausfällt.

\section{Nachtlauf im Englischen Garten}

Der Startschuss für den Lauf fällt in den Abendstunden des 27. März 2019 direkt beim Chinesischen Turm. Von dort aus führt der 2,5 Kilometer lange autofreie und rollstuhlgeeignete Rundkurs durch den Englischen Garten. Die Teilnehmer können eine oder zwei Runden laufen bzw. walken; für
Läufer sind auch vier Runden, also zehn Kilometer möglich. Rund um den Lauf ist ein abwechslungsreiches Programm vorgesehen: Prominente Persönlichkeiten aus Politik, Sport und Gesellschaft sind ebenso vor Ort wie mehrere Musikgruppen. TV-Moderator Jörg Wontorra führt durch den Abend.

\section{Spende für soziale Organisationen Die Startgebühr beträgt 20 Euro. Davon gehen acht Euro als Spende an drei verschie- dene Projekte: Die Kinderhilfe Organtrans- plantation und das Rehabilitationszentrum Ederhof helfen Kindern und deren Fami- lien vor und nach einer Transplantation. Der Joachim-Deckarm-Fonds der Deutschen Sporthilfe unterstützt den früheren Hand- ball-Nationalspieler, der bei einem Unfall während eines Europapokalspiels 1979 ein schweres Schädel-Hirn-Trauma erlitt und seitdem auf fremde Hilfe angewiesen ist.}

Quelle:DGCH 